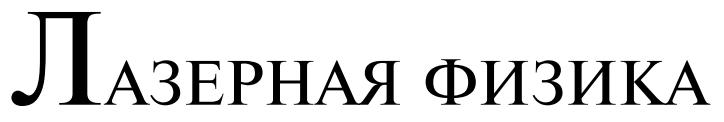

\section{LASER PHYSICS}

\section{РЕЖИМЫ ОБРАБОТКИ ПОАЛОЖЕК И НАНЕСЕНИЯ НАНОПОКРЫТИЙ С ПОМОЩЬЮ МАЗЕРНО-ПААЗМЕННОГО МЕТОАА}

\author{
В. К. ГОНЧАРОВ ${ }^{1)}$, М. В. ПУЗЫРЕВ ${ }^{1)}$, \\ Д. П. ПРОКОПЕНЯ ${ }^{1)}$, Н. И. ШУЛЬГАН ${ }^{2)}$ В. Ю. СТУПАКЕВИЧ \\ ${ }^{1)}$ Институт прикладных физических проблем им. А. Н. Севченко БГУ, \\ ул. Курчатова, 7, 220045, г. Минск, Беларусь \\ ${ }^{2)}$ Белорусский государственный университет, пр. Независимости, 4, 220030, г. Минск, Беларусь \\ ${ }^{3)}$ Гродненский государственный университет им. Янки Купаль, \\ ул. Ожешко, 22, 230023, г. Гродно, Беларусь
}

Изучены физические процессы в лазерно-плазменном источнике, который используется для нанесения наноструктур и представляет собой эрозионный лазерный факел материала мишени и подложку, расположенную в вакуумной камере. Для плавной регулировки параметров наносимых на подложку частиц между лазерной мишенью и подложкой предложено поместить сетку, на которую подается отрицательный потенциал по отношению к лазерной

\section{Образец цитирования:}

Гончаров ВК, Пузырев МВ, Прокопеня ДП, Шульган НИ, Ступакевич ВЮ. Режимы обработки подложек и нанесения нанопокрытий с помощью лазерно-плазменного метода. Журнал Белорусского государственного университета. Физика. 2021;1:73-81.

https://doi.org/10.33581/2520-2243-2021-1-73-81

\section{For citation:}

Goncharov VK, Puzyrev MV, Prakapenia DP, Shulhan NI, Stupakevich VYu. Regimes of substrates processing and deposition nanofilms using the laser-plasma method. Journal of the Belarusian State University. Physics. 2021;1:73-81. Russian. https://doi.org/10.33581/2520-2243-2021-1-73-81

\begin{abstract}
Ав торы:
Виктор Константинович Гончаров - доктор физико-математических наук, профессор; главный научный сотрудник лаборатории лазерной плазмодинамики.

Михаил Валентинович Пузырев - кандидат физико-математических наук, доцент; заведующий лабораторией лазерной плазмодинамики.

Дмитрий Павлович Прокопеня - младший научный сотрудник лаборатории лазерной плазмодинамики.

Никита Игоревич Шульган - студент факультета радиофизики и компьютерных технологий. Научный руководитель - В. К. Гончаров.

Валерий Юзефович Ступакевич - старший преподаватель кафедры информационных систем и технологий физикотехнического факультета.
\end{abstract}

Authors:

Victor K. Goncharov, doctor of science (physics and mathematics), full professor; leading researcher at the laboratory of laser plasma dynamics.

Michail V. Puzyrev, PhD (physics and mathematics), docent; head of the laboratory of laser plasma dynamics.

puzyrev@bsu.by

Dzmitry P. Prakapenia, junior researcher at the laboratory of laser plasma dynamics.

dmitry.prokopenia@gmail.com

Nikita I. Shulhan, student at the faculty of radiophysics and computer technologies.

nikita.shulgan@gmail.com

Valery Yu. Stupakevich, senior lecturer at the department of information systems and technologies, physico-technical faculty. tv_sad@grsu.by 
мишени. В результате после сетки формируется поток частиц, состоящий преимущественно из ионов, энергией которых можно надежно и плавно управлять, подавая на сетку положительный потенциал по отношению к подложке. Экспериментально обоснован метод нанесения нанопокрытий с помощью ионов из лазерной плазмы. Показано, что в лазерно-плазменном источнике для нанесения наноструктур можно реализовать различные режимы обработки поверхности подложки. Данный источник позволяет последовательно, не разгерметизируя вакуумную камеру, произвести очистку поверхности подложки, создать псевдодиффузионный слой материала лазерной мишени в приповерхностном слое подложки. Это обеспечит получение высокоадгезионных нанопокрытий с заранее заданными параметрами.

Ключевые слова: лазерная плазма; ионные пучки; наноструктуры; высокая адгезия.

\title{
REGIMES OF SUBSTRATES PROCESSING AND DEPOSITION NANOFILMS USING THE LASER-PLASMA METHOD
}

\author{
V. K. GONCHAROV ${ }^{\mathrm{a}}$, M. V.PUZYREV ${ }^{\mathrm{a}}$, \\ D. P. PRAKAPENIA ${ }^{\mathrm{a}}$, N. I. SHULHAN ${ }^{\mathrm{b}}$, V. YU. STUPAKEVICH \\ ${ }^{a}$ A. N. Sevchenko Institute of Applied Physical Problems, Belarusian State University, \\ 7 Kurčatava Street, Minsk 220045, Belarus \\ ${ }^{\mathrm{b}}$ Belarusian State University, 4 Niezaliežnasci Avenue, Minsk 220030, Belarus \\ ${ }^{c}$ Yanka Kupala State University of Grodno, 22 Ažeška Street, Hrodna 230023, Belarus \\ Corresponding author: M. V. Puzyrev (puzyrev@bsu.by)
}

The physical processes occurring in a laser-plasma source is used for deposition nanostructures. The laser-plasma source is an erosion laser plume of the target material and a substrate located in a vacuum chamber. It has been proposed to place a grid between the laser target and the substrate. A negative potential is applied to the grid relative to the laser target to smoothly adjust the parameters of the particles deposited on the substrate. As a result, a particles flow is formed after a grid. This particle flow is predominantly consisting of ions. The energy of the ions can be reliably and smoothly controlled by applying a positive potential to the grid relative to the substrate. It has been experimentally proved method for deposition of nanofilms using ion beams from the laser plasma. It has been shown that different regimes of substrate surface treatment can be implemented in the laser-plasma source for deposition nanostructures. Using this source, you can sequentially clean the surface of the substrate without depressurizing the vacuum chamber, and create a pseudodiffusion layer of the laser target material near the surface layer of the substrate. It will allow producing it possible to obtain highly adhesive nanofilms with predetermined parameters.

Keywords: laser plasma; ion beams; nanostructures; high adhesion.

\section{Введение}

Метод лазерно-плазменного нанесения нанопокрытий обладает рядом преимуществ. Прежде всего к ним относят возможность получения плазмы из любого вещества в любом агрегатном состоянии и стерильность.

Физические явления, происходящие при воздействии мощного лазерного излучения на вещества (нагрев, плавление, испарение и плазмообразование), описаны в монографиях [1-3].

Для нанесения нанопокрытий достаточно воздействовать лазерным излучением на мишень, и продукты разрушения в виде плазмы, распространяясь перпендикулярно к поверхности мишени, достигают поверхности подложки, расположенной на некотором расстоянии от мишени параллельно последней [4; 5].

Однако у этого метода есть и недостатки, в частности довольно сложные устройства плавной регулировки параметров при автоматизации режимов нанесения нанопленок.

В целях устранения указанной проблемы в работе [6] было предложено получать из лазерной плазмы поток ионов и плавно регулировать энергию ионов и плотность ионного потока путем плавного изменения потенциала электрического поля. Электрическая схема таких экспериментов представлена на рис. 1. 


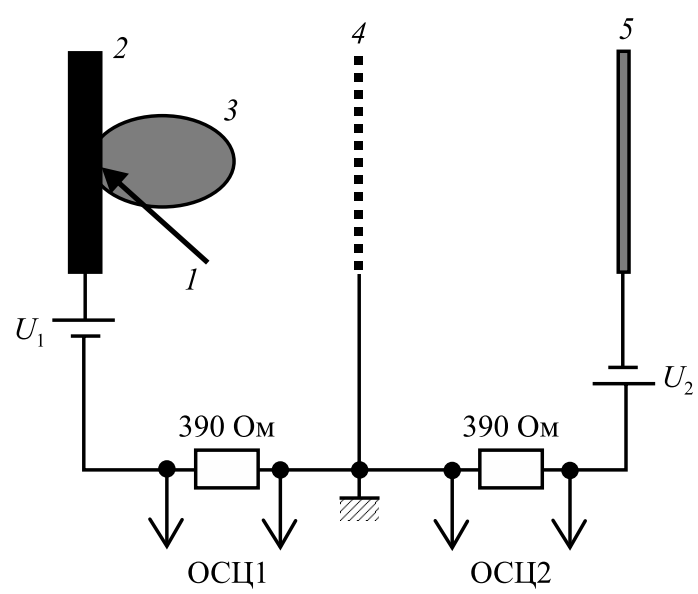

Puc. 1. Электрическая схема эксперимента:

1 - лазерное излучение; 2 - лазерная мишень; 3 - эрозионный плазменный факел; 4 - сетка; 5 - подложка; ОСЦ1 и ОСЦ2 - сигналы, снимаемые на первый и второй каналы осциллографа с сопротивлений нагрузки 390 Ом;

$U_{1}$ и $U_{2}-$ независимые источники питания

Fig. 1. Electrical scheme of the experiment:

1 - laser radiation; 2 - laser target; 3 - erosive plasma plume; 4 - grid; 5 - substrate; OSC1 and OSC2 - signals taken on the first and second channels of the oscilloscope with resistances of $390 \Omega ; U_{1}$ and $U_{2}$-independent power sources

\section{Экспериментальное оборудование и методики экспериментов}

Мишень подвергалась воздействию излучения лазера Nd:YAG LS-2137 (LOTIS TII, Беларусь - Япония). Измерения производились с помощью осциллографа TDS2022B (Tektronix, США). Воздействие лазерного излучения реализовывалось при различных плотностях мощности. При этом на промежутки мишень - сетка и сетка - подложка подавались разные потенциалы $U_{1}$ и $U_{2}$.

Так как в экспериментах присутствуют два источника питания, важно определить, в какой точке производить заземление. Предварительно были проведены эксперименты с заземлением лазерной мишени и подложки. Оказалось, что в данных случаях источники питания через внутреннее сопротивление участков мишень - сетка и сетка - подложка влияют друг на друга. И только схема с заземленной сеткой (см. рис. 1) позволяет источникам питания $U_{1}$ и $U_{2}$ оставаться независимыми при любых режимах экспериментов.

\section{Результаты и их обсуждение}

В настоящей работе в качестве мишеней использовались медь, алюминий, серебро, графит при разных сочетаниях материала подложки. Так же как и в статье [6] для алюминиевой мишени, для мишеней из меди, серебра и графита токовые характеристики в промежутке мишень - сетка имеют несколько «горбов», которые отличаются только интенсивностью и длительностью, что связано с различием оптофизических характеристик материалов мишени (рис. 2).

Кривые, изображенные на рис. 2, можно объяснить следующим образом. При появлении плазмы в промежутке мишень - сетка подача на сетку потенциала $\sim 5$ В по отношению к мишени заставляет без организации двойного слоя ионы двигаться к отрицательному потенциалу сетки, а электроны - к мишени. Однако из-за экранирования потенциала сетки ионами электроны медленно двигаются к сетке за счет динамики продуктов разрушения или совсем с небольшой скоростью - к поверхности мишени. В это время ток в цепи сетка - мишень определяется в основном током ионов (первый «горб»).

Как только основной поток ионов пролетел сетку ( 4 мкс после начала воздействия лазерного излучения), потенциал, поданный на нее с источника питания $U_{1}$, уже не экранирован ионами, и электроны начинают двигаться в сторону мишени, резко увеличивая обратный ток электронов (второй «горб»).

Третий «горб» на кривой тока в промежутке мишень - сетка определяется прямым ионным потоком и обратным потоком электронов в конце распадающегося лазерно-плазменного факела с уменьшающимися параметрами.

Как показали эксперименты [6-8], при подаче на сетку отрицательного потенциала 5 В по отношению к мишени после сетки формируется поток заряженных частиц, состоящий преимущественно из ионов. Управление энергией ионов и плотностью их потока легко осуществляется подачей на сетку положительного потенциала по отношению к подложке. В режиме лазерно-плазменного источника ионов с заземленной сеткой процессы, происходящие в промежутке сетка - подложка, не влияют на процессы в промежутке мишень - сетка. 

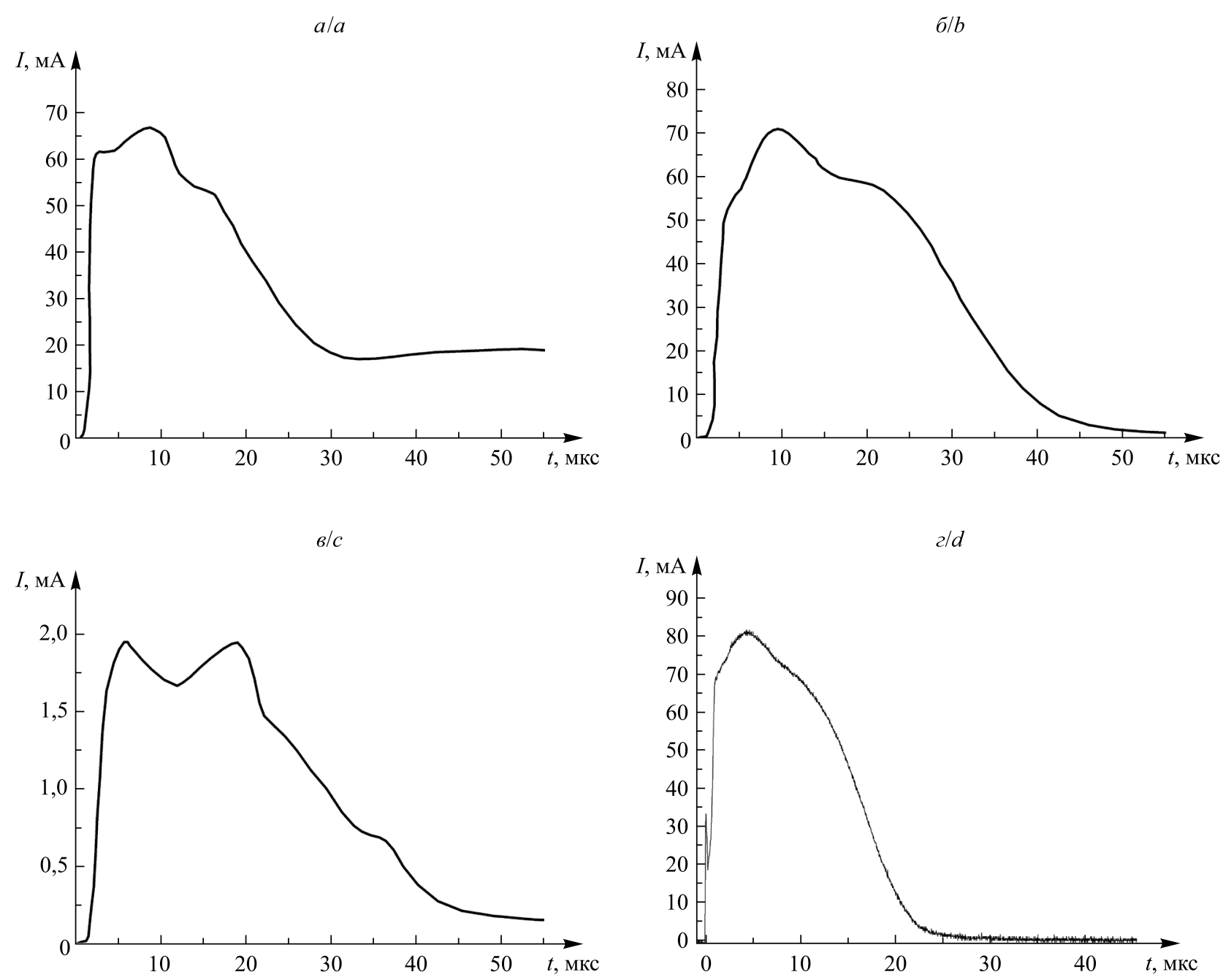

Puc. 2. Ток в промежутке мишень - сетка при воздействии лазерного излучения на мишени из различных материалов: $a$ - алюминиевая мишень; $\sigma$ - медная мишень; в - углеродная мишень; 2 - серебряная мишень

Fig. 2. Current in the target - grid interval at acting laser radiation on various materials targets: $a$ - aluminum target; $b$ - copper target; $c$ - carbon target; $d$ - silver target

Рассмотрим различные варианты режимов источника при изменении плотности мощности воздействующего на мишень лазерного излучения, материала мишени и подложки, а также потенциала в промежутке сетка - подложка. Потенциал в промежутке мишень - сетка при этом устанавливают таким, чтобы после сетки поток заряженных частиц состоял преимущественно из ионов.

При воздействии на мишень лазерного излучения с различной плотностью мощности в эрозионном лазерном факеле образуется плазма с разными температурой, давлением и, как следствие, кинетической энергией электронов и ионов. Поэтому даже без подачи потенциала на подложку в промежутке сетка - подложка формируется поток ионов с различными первоначальной энергией и плотностью потока. Данный эффект заметен и при изменении потенциала в промежутке сетка - подложка. На рис. 3 представлены кривые тока ионов на подложке из кремния марки КДБ03 при различных плотностях мощности воздействующего на медную мишень лазерного излучения, а также разных потенциалах электрического поля в промежутке сетка - подложка. При этом на промежуток мишень - сетка подавался потенциал, при котором после сетки формировался ток, состоящий преимущественно из ионов.

Как видно из рис. 3 , при малых плотностях мощности лазерного излучения на подложку поступает ионный поток, по форме близкий к плазменному потоку из мишени (см. рис. 3 , кривая 1). При увеличении плотности мощности лазерного излучения (см. рис. 3, кривая 2) на подложку поступает больший по абсолютной интенсивности ионный поток, ограниченный на вершине импульса. Это объясняется тем, что за счет кинетической энергии плазменного потока и дополнительного ускорения электрическим полем энергия ионов становится достаточной для того, чтобы на подложке сформировался поток вторичных ионов, движущихся в противоположную сторону. 


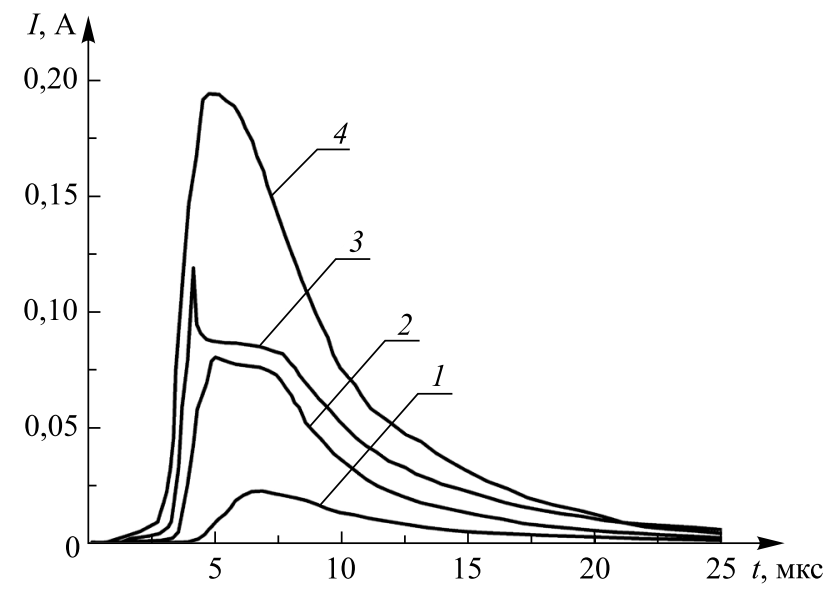

Puc. 3. Ток на кремниевой подложке КДБ03 при различных плотностях мощности воздействующего на медную мишень лазерного излучения и разных потенциалах в промежутке сетка - подложка (на участке мишень - сетка установлен постоянный потенциал $U_{1}=30 \mathrm{~B}$ ):

$$
\begin{aligned}
& 1-1,5 \cdot 10^{9} \mathrm{BT} / \mathrm{cm}^{2}, U_{2}=50 \mathrm{~B} ; 2-1,7 \cdot 10^{9} \mathrm{BT} / \mathrm{cm}^{2}, U_{2}=50 \mathrm{~B} ; \\
& 3-1,8 \cdot 10^{9} \mathrm{BT} / \mathrm{cm}^{2}, U_{2}=50 \mathrm{~B} ; 4-1,8 \cdot 10^{9} \mathrm{BT} / \mathrm{cm}^{2}, U_{2}=100 \mathrm{~B}
\end{aligned}
$$

Fig. 3. Current on a silicon substrate KDB03 at acting laser radiation on a copper target at different power densities and at different potentials in the grid - substrate interval (the interval target - grid have constant potential $U_{1}=30 \mathrm{~V}$ ): $1-1.5 \cdot 10^{9} \mathrm{~W} / \mathrm{cm}^{2}, U_{2}=50 \mathrm{~V}$; $2-1.7 \cdot 10^{9} \mathrm{~W} / \mathrm{cm}^{2}, U_{2}=50 \mathrm{~V} ; 3-1.8 \cdot 10^{9} \mathrm{~W} / \mathrm{cm}^{2}, U_{2}=50 \mathrm{~V} ; 4-1.8 \cdot 10^{9} \mathrm{~W} / \mathrm{cm}^{2}, U_{2}=100 \mathrm{~V}$

При дальнейшем увеличении плотности мощности лазерного излучения ограничение вершины импульса ионного тока усиливается из-за увеличения первоначальной кинетической энергии ионов вследствие газодинамического ускорения (см. рис. 3, кривая 3).

Однако дальнейший рост кинетической энергии ионов за счет электрического поля в промежутке сетка - подложка приводит к тому, что временная форма импульса ионного тока повторяет временную форму плазменного факела и не имеет ограничений на вершине импульса (см. рис. 3, кривая 4). Явление связано с тем, что кинетическая энергия ионов достигает такой величины, при которой сечение столкновения с ионами подложки уменьшается, вследствие чего уменьшается и вторичная эмиссия, а ионы материала лазерной мишени имплантируются в материал подложки. При этом интенсивность ионного потока значительно возрастает (см. рис. 3, кривая 4).

Как показали эксперименты, при разнообразных изменениях условий в промежутке сетка - подложка происходящие в нем процессы не влияют на процессы в промежутке мишень - сетка ввиду того, что мы использовали вариант с заземленной сеткой. Учитывая этот факт, можно более детально исследовать процессы в промежутке сетка - подложка при определенных постоянных условиях в промежутке мишень - сетка.

Как видно по кривым 2 и 3 на рис. 3, при таких условиях возникают вторичная эмиссия ионов с подложки и ограничение вершины импульса тока ионов в цепи сетка - подложка. В данном случае реализуется режим травления поверхности подложки падающим ионным потоком. Поскольку этот режим имеет большое значение при нанесении высокоадгезионных покрытий, рассмотрим его более подробно.

На рис. $4, a$, изображен общий вид кривой тока в цепи сетка - подложка в режиме травления подложки потоком первичных ионов. На переднем фронте импульса имеются несколько пиков, затем при появлении вторичной эмиссии за счет противоположного потока вторичных ионов наблюдается достаточно плоская вершина, а когда вторичная эмиссия пропадает, импульс ионного тока в цепи сетка - подложка по экспоненте падает до нуля, следуя по временной форме за формой плазменного факела в промежутке мишень - сетка. На рис. 4, $\sigma-2$, с высоким временным разрешением представлены передние фронты импульса тока в промежутке сетка - подложка при различных условиях.

Первый пик тока в промежутке сетка - подложка (см. рис. 4, б) обусловлен обратным током электронов с подложки за счет фотоэффекта при облучении поверхности подложки отраженной от лазерной мишени частью воздействующего лазерного излучения. Через 1,5 мкс появляется второй пик в цепи сетка - подложка. Исходя из скоростей плазменного факела $(\sim 10$ км/с), данный результат можно объяснить образованием вторичной плазмы из материала сетки за счет воздействия на последнюю лазерного излучения, отраженного от мишени, и излучения плазмы вблизи поверхности мишени. Однако эти процессы незначительно влияют на основной ток в цепи сетка - подложка.

Через 3,8 мкс появляется основной пик тока в промежутке сетка - подложка. Его длительность составляет 250 нс по полувысоте, после чего наблюдается ограничение импульса за счет вторичной эмиссии (см. рис. $4, \sigma$ ). 
При увеличении потенциала на промежутке сетка - подложка (см. рис. 4 , в) максимум пика появляется раньше - через 3,5 мкс, что говорит об увеличении скорости потока заряженных частиц за счет ускорения электрическим полем. При увеличении плотности мощности воздействующего на мишень лазерного излучения скорость процессов в промежутке сетка - подложка растет, и максимум пика появляется еще раньше - через 3,2 мкс (см. рис. 4,2 ).

Представленные на рис. 4 результаты экспериментов, в которых перед появлением ограничения импульса тока в промежутке сетка - подложка наблюдается короткий (порядка 250 нс) положительный импульс тока на подложку, можно объяснить следующим образом. В самом начале процесса поступления заряженных частиц на подложку попадают наиболее быстрые ионы, энергии которых хватает для их имплантации в материал подложки. С течением времени на подложку поступают все более медленные ионы, и наступает момент, когда механизм имплантации меняется на механизм травления, т. е. вторичной эмиссии ионов. Однако при имплантации в приповерхностном слое подложки из-за наличия ионов формируется (накапливается) положительный заряд. Для его компенсации в цепи сетка - подложка за счет источника $U_{2}$ появляется обратный ток электронов, который должен иметь на осциллограмме положительный импульс. Частично импульс электронов компенсирует заряд, созданный внедренными ионами, а дальше возникает вторичная эмиссия, которая вследствие максимального значения вторичного ионного тока в это время является преобладающим процессом, и в импульсе тока на подложку появляется ограничение в виде полки различной длительности в зависимости от условий эксперимента.

$a / a$

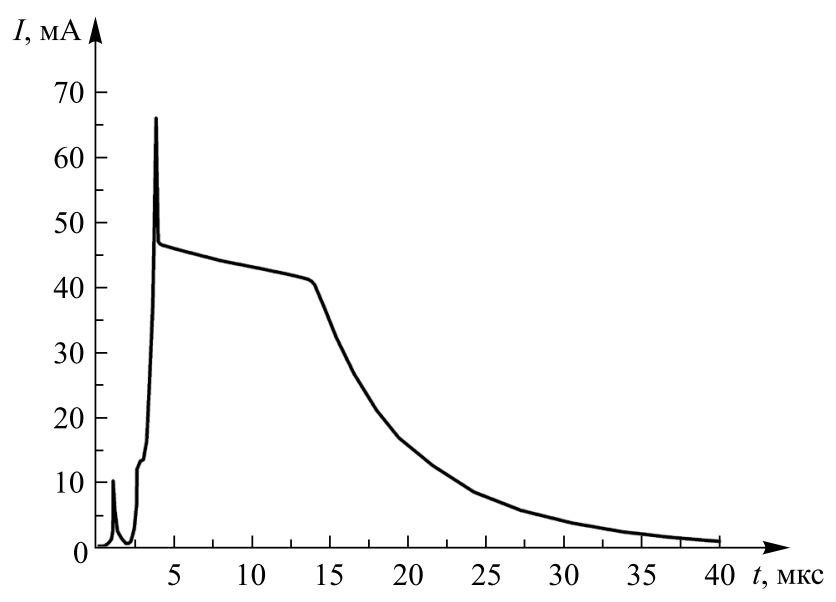

$B / c$

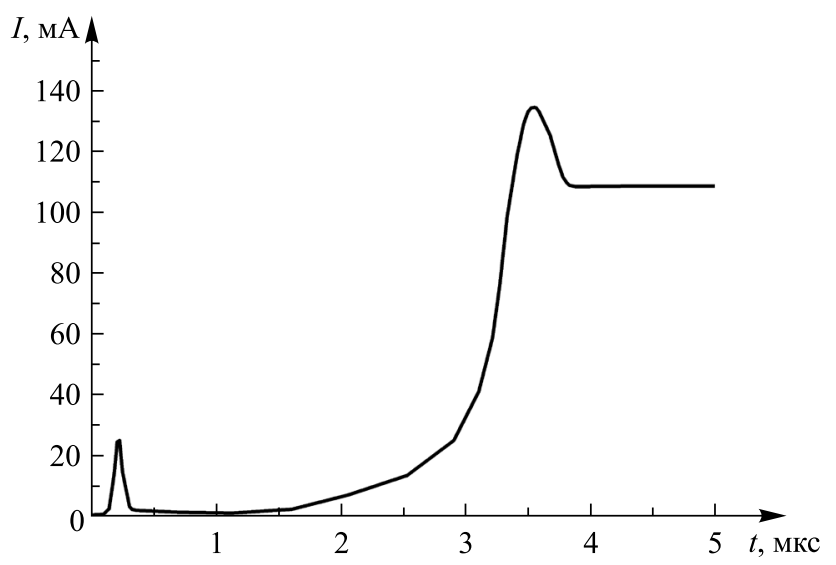

$\sigma / b$

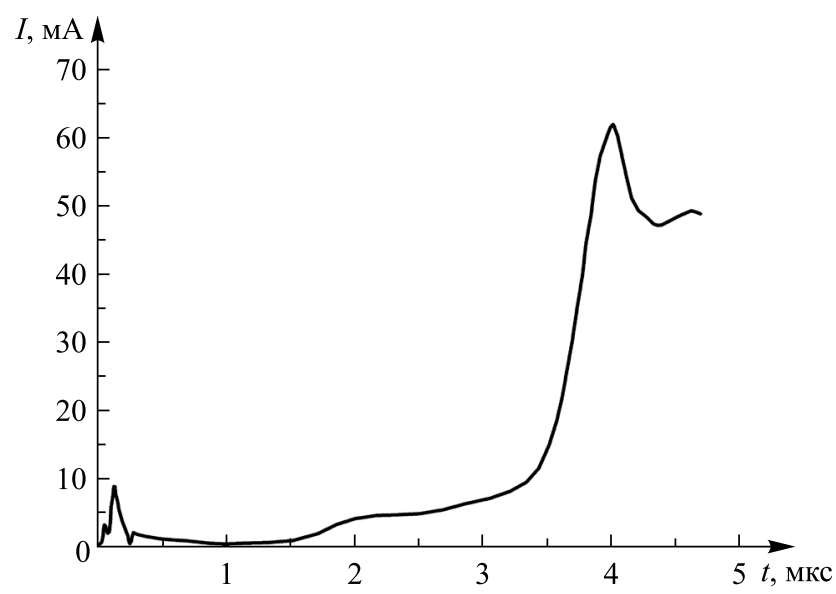

2/d

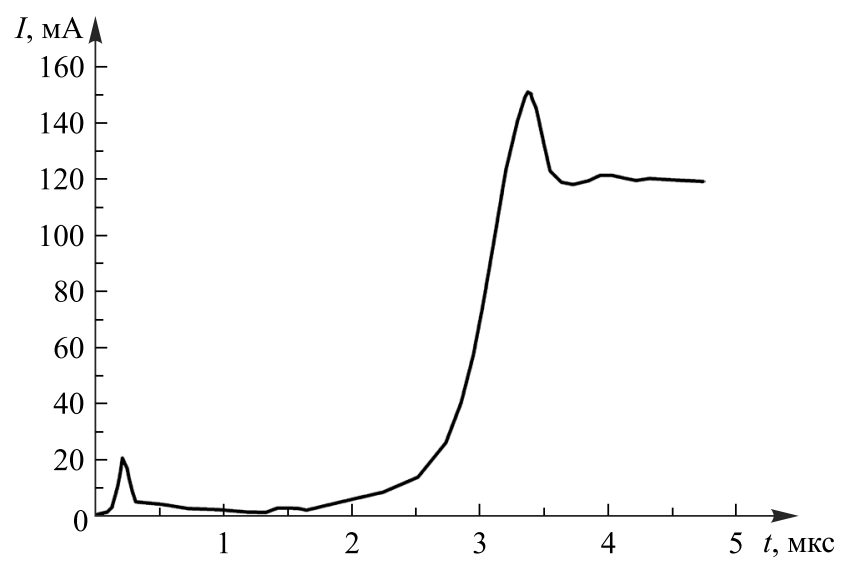

Puc. 4. Общий импульс тока и его передний фронт в промежутке сетка - подложка в режиме вторичной ионной эмиссии при различных условиях воздействия лазерного излучения на медную мишень (на участке мишень - сетка установлен постоянный потенциал $U_{1}=30 \mathrm{~B}$ ): $a, \sigma$ - плотность мощности лазерного излучения $5 \cdot 10^{8} \mathrm{BT} / \mathrm{cm}^{2}, U_{2}=30 \mathrm{~B}$; $b$ - плотность мощности лазерного излучения $5 \cdot 10^{8} \mathrm{BT} / \mathrm{cm}^{2}, U_{2}=50 \mathrm{~B}$; 2 - плотность мощности лазерного излучения $1 \cdot 10^{9} \mathrm{BT} / \mathrm{cm}^{2}, U_{2}=50 \mathrm{~B}$

Fig. 4. Total current pulse and its leading edge in the grid - substrate interval in the regime secondary ion emission at the different conditions of the acting laser radiation on a copper target

(the interval target - grid have constant potential $U_{1}=30 \mathrm{~V}$ ): $a, b$ - laser power density $5 \cdot 10^{8} \mathrm{~W} / \mathrm{cm}^{2}, U_{2}=30 \mathrm{~V}$; $c$ - laser power density $5 \cdot 10^{8} \mathrm{~W} / \mathrm{cm}^{2}, U_{2}=50 \mathrm{~V} ; d$ - laser power density $1 \cdot 10^{9} \mathrm{~W} / \mathrm{cm}^{2}, U_{2}=50 \mathrm{~V}$ 
Для подтверждения режима травления подложки (вторичной ионной эмиссии) были поставлены эксперименты с потоками различных ионов ( $\mathrm{Al}, \mathrm{Ag}, \mathrm{Cu})$ на подложки из разных материалов (КДБ03, КЭФ20, 08Х18Н10Т) с постоянными потенциалами в промежутках мишень - сетка (20 В) и сетка - подложка (50 В). Результаты экспериментов приведены на рис. 5.

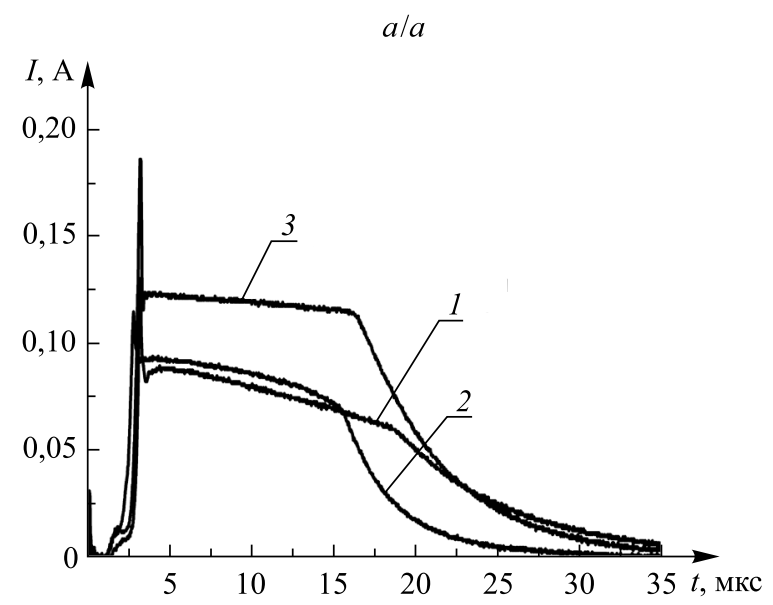

$\sigma / b$

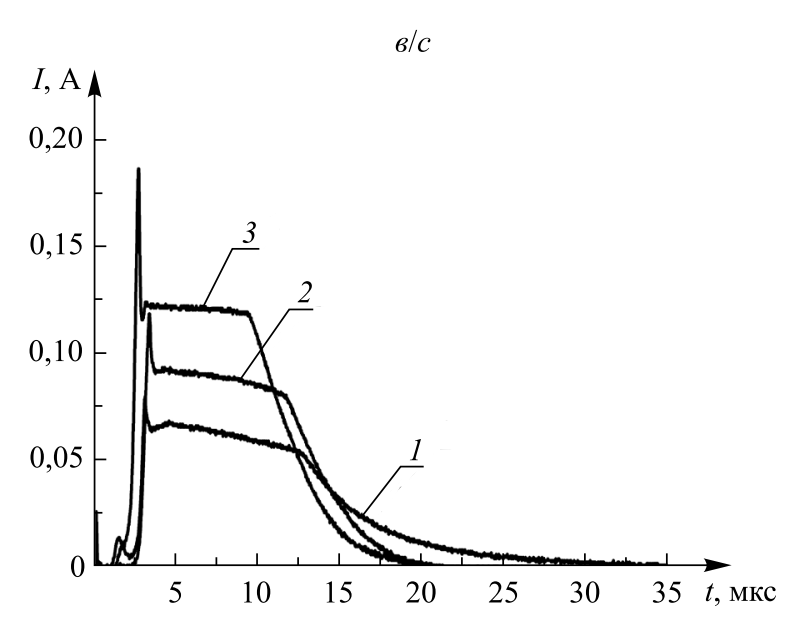

Puc. 5. Ток на подложке при воздействии лазерного излучения с плотностью мощности $1,8 \cdot 10^{9} \mathrm{BT} / \mathrm{cm}^{2}$ на различные мишени с постоянными потенциалами в промежутках мишень - сетка (20 В) и сетка - подложка (50 B): $a-\mathrm{Al} ; \sigma-\mathrm{Ag} ;$ в - Cu. Материалы подложки: 1 - КДБ03; 2 - КЭФ20; 3 - 08Х18Н10Т

Fig. 5. Current on the at acting laser radiation with a power density of $1.8 \cdot 10^{9} \mathrm{~W} / \mathrm{cm}^{2}$ on various targets with constant potentials in the intervals target - grid $(20 \mathrm{~V})$ and grid - substrate $(50 \mathrm{~V})$ : $a-\mathrm{Al} ; b-\mathrm{Ag} ; c-\mathrm{Cu}$. Substrate materials: $1-\mathrm{KDB} 03 ; 2-\mathrm{KEF} 20 ; 3-08 \mathrm{H} 18 \mathrm{~N} 10 \mathrm{~T}$

Как видно на рис. 5, во всех случаях наблюдается короткий пичок (250 нс) на импульсе тока в промежутке сетка - подложка за счет обратного тока электронов, компенсирующего заряд имплантированных в приповерхностную область подложки ионов, с помощью источника $U_{2}$.

Кроме того, результаты этих экспериментов показывают (см. рис. 5), что значения тока в промежутке сетка - подложка зависят как от материала лазерной мишени, так и от материала подложки.

Из-за того, что материалы мишени имеют разный коэффициент поглощения на длине волны лазерного излучения (1,06 мкм), в промежутке мишень - сетка образуется плазма с различными параметрами (температура, давление, концентрация заряженных частиц, длительность существования).

Из используемых в экспериментах материалов минимальным коэффициентом поглощения обладает медь, у серебра коэффициент поглощения несколько выше, максимальный же коэффициент поглощения имеет алюминий. Этим и объясняется разница в величинах тока и длительности импульса в промежутке сетка - подложка (см. рис. 5).

Для выяснения влияния материала подложки на форму импульса тока в промежутке сетка - подложка были взяты наиболее используемые в наших экспериментах материалы подложек, удобные для исследования на электронном микроскопе и отличающиеся по проводимости и атомному весу. 
Как видно из рис. 5, $a$, количественное значение тока в промежутке сетка - подложка зависит от материала подложки. Наименьшая амплитуда импульса тока отмечена у подложки из кремния марки КДБ03. В данном случае первичные ионы алюминия более эффективно выбивают из поверхности подложки ионы бора, и вторичный ток ионов максимально компенсирует первичный ионный ток. Поэтому амплитуда импульса в промежутке минимальна. Для подложки из кремния марки КЭФ20 из-за уменьшения соотношения атомных весов алюминия и фосфора по сравнению с соотношением атомов алюминия и бора происходит уменьшение тока вторичной эмиссии. Вследствие этого увеличиваются разница между первичным и вторичным токами ионов и импульс общего тока в промежутке сетка подложка (см. рис. 5, $a$, кривые 1 и 2).

При использовании подложки из нержавеющей стали марки 08X18Н10Т эффект усиливается, так как атомный вес хрома (самый легкий по атомному весу элемент сплава 08Х18Н10Т) больше атомного веса алюминия. Вторичная эмиссия в данном случае еще меньше. Вследствие этого импульс общего тока в промежутке сетка - подложка (см. рис. 5, $a$, кривая 3) существенно увеличивает свое значение (примерно в 1,5 раза).

При использовании серебряной мишени из-за большей массы первичных ионов серебра вторичная эмиссия с поверхности подложек увеличивается, уменьшая при этом величину общего тока в цепи сетка подложка (см. рис. 5, б).

Качественно картина повторяется и в экспериментах с медной мишенью. Однако значения токов в этом случае определяются не только соотношением атомных масс первичных и вторичных ионов, но и существенным влиянием коэффициента поглощения лазерного излучения материалом мишени.

Из всех рассматриваемых в настоящей работе материалов лазерной мишени медь обладает наименьшим коэффициентом поглощения на длине волны лазерного излучения (1,06 мкм). Поэтому эффективность взаимодействия лазерного излучения с медной мишенью существенно ниже, чем с алюминиевой и серебряной мишенями. Так как форма лазерного импульса представляет собой асимметричный колокол, то и время плазмообразования уменьшается, и параметры плазмы (температура, концентрация заряженных частиц) понижаются. За счет этого количественные значения импульса тока в промежутке сетка - подложка ниже, чем при использовании серебряной мишени, кроме того, уменьшается длительность импульса.

\section{Заключение}

Таким образом, для лазерно-плазменного источника напыления нанопокрытий на основе экспериментального изучения явлений в промежутках мишень - сетка и сетка - подложка для различных материалов мишени и подложки объяснены процессы, происходящие при управлении электрическим полем лазерной плазмы в вакууме.

Экспериментально установлено, что при заземленной сетке источники питания промежутков мишень - сетка и сетка - подложка независимы друг от друга при любых режимах эксперимента.

Путем использования различных материалов мишени и подложки и изменения условий эксперимента продемонстрировано, что процессы, происходящие в промежутке сетка - подложка, при заземленной сетке не влияют на процессы в промежутке мишень - сетка.

Объяснена форма тока в промежутке сетка - подложка. Показано, что влияние фотоионизации и вторичной плазмы материала сетки незначительно.

Отмечено, что при травлении ионными потоками поверхности подложки появление короткого пика тока перед началом вторичной ионной эмиссии практически не сказывается на переносе массы материала мишени и подложки, так как он возникает за счет обратного тока электронов, компенсирующего положительный заряд ионов, имплантированных в поверхность подложки.

Экспериментально показано, что на процессы травления влияют характеристики как материала лазерной мишени, так и материала подложки.

В настоящей работе рассмотрены режимы травления поверхности подложки, имплантации ионов материала лазерной мишени в приповерхностную область подложки и нанесения материала мишени на подложку последовательно без разгерметизации вакуумной камеры. Это позволит с помощью лазерноплазменного метода получать высокоадгезионные нанопокрытия.

\section{Библиографические ссылки}

1. Анисимов СИ, Имас ЯА, Романов ГС, Ходыко ЮВ. Действие излучения большой мощңности на металл. Москва: Наука; 1970. $272 \mathrm{c}$.

2. Реди Дж. Действие мощного лазерного излучения. Москва: Мир; 1974. 468 с.

3. Goncharov VK, Kozadaev KV, Puzyrev MV. The influence of ND laser irradiation parameters on dynamics of metal condensed phase propagating near target. In: Sosa M, Franco J, editors. Engineering physics and mechanics. Analyses, prediction and applications. New York: Nova Science Publishers; 2010. p. 441-471. (Engineering tools, techniques and tables series). 
4. Chrisey DB, Hubler GK. Pulsed lased deposition of thin films. New York: Wiley-Interscience; 1994. 648 p.

5. Bonelli M, Miotello A, Mosaner P. Pulsed laser deposition of diamondlike carbon films on polycarbonate. Journal of Applied Physics. 2003;93:859-865. DOI: 10.1063/1.1530725.

6. Гончаров ВК, Пузырев МВ, Ступакевич ВЮ. Физические процессы в лазерном источнике ионов алюминия с управляемой энергией для нанесения нанопленок. Журнал Белорусского государственного университета. Физика. 2017;3:79-87.

7. Гончаров ВК, Василевич АЕ, Ступакевич ВЮ, Пузырев МВ. Лазерно-плазменный источник ионов с регулируемой энергией для нанесения нанопленок. Электроника инфо. 2016;11:54-57.

8. Гончаров ВК, Пузырев МВ, Ступакевич ВЮ. Управление потоками заряженных частиц в эрозионной лазерной плазме графитовой мишени в вакууме. Инженерно-физический журнал. 2018;91(4):1115-1121.

\section{References}

1. Anisimov SI, Imas YaA, Romanov GS, Khodyko YuV. Deistvie izlucheniya bol'shoi moshchnosti na metall [The effect of highpower radiation on metal]. Moscow: Nauka; 1970. 272 p. Russian.

2. Redi J. Deistvie moshchnogo lazernogo izlucheniya [The action of powerful laser radiation]. Moscow: Mir; 1974. 468 p. Russian.

3. Goncharov VK, Kozadaev KV, Puzyrev MV. The influence of ND laser irradiation parameters on dynamics of metal condensed phase propagating near target. In: Sosa M, Franco J, editors. Engineering physics and mechanics. Analyses, prediction and applications. New York: Nova Science Publishers; 2010. p. 441-471. (Engineering tools, techniques and tables series).

4. Chrisey DB, Hubler GK. Pulsed lased deposition of thin films. New York: Wiley-Interscience; 1994. 648 p.

5. Bonelli M, Miotello A, Mosaner P. Pulsed laser deposition of diamondlike carbon films on polycarbonate. Journal of Applied Physics. 2003;93:859-865. DOI: 10.1063/1.1530725.

6. Goncharov VK, Puzyrev MV, Stupakevich VY. Physical processes in a laser source of aluminum ions with the controlled energy for nanofilm deposition. Journal of the Belarusian State University. Physics. 2017;3:79-87. Russian.

7. Goncharov VK, Vasilevich AE, Stupakevich VYu, Puzyrev MV. [Laser-plasma ion source with controlled energy for nanofilm deposition]. Elektronika info. 2016;11:54-57. Russian.

8. Goncharov VK, Puzyrev MV, Stupakevich VYu. [Controling charged particle fluxes in the erosive laser plasma of a graphite target in vacuum]. Inzhenerno-fizicheskii zhurnal. 2018;91(4):1115-1121. Russian. 